\title{
ESTILOS DE LIDERAZGO, RIESGO PSICOSOCIAL Y CLIMA ORGANIZACIONAL EN UN GRUPO DE EMPRESAS COLOMBIANAS
}

\author{
FRANCOISE CONTRERAS ${ }^{* *}$, FERNANDO JUÁREZ $Z^{* * *}$, DAVID BARBOSA ${ }^{* * * *} \&$ \\ ANA FERNANDA URIBE \\ UNIVERSIDAD DEL ROSARIO - UNIVERSIDAD PONTIFICIA BOLIVARIANA (BUCARAMANGA)
}

Recibido/Received/Recebido: 16/04/2010 - Aceptado/Accepted/Aprovado: 06/10/2010

\begin{abstract}
Resumen
Se analizaron las relaciones entre los riesgos psicosociales, el clima organizacional y el estilo de liderazgo, en empresas colombianas del sector de servicios sociales y de salud. Cuatrocientos participantes respondieron la Escala de Clima Organizacional (ECO), el Cuestionario de Factores Psicosociales en el Trabajo (CFP) y el Test de Adjetivos de Pitcher (Pitcher Test Adjectives [PAT $\neg$ ]). Se obtuvieron coeficientes de correlación y regresiones lineales. En general, las dimensiones del ECO correlacionaron negativamente con las del CFP. En el PAT se obtuvieron dos estilos de liderazgo, uno deseable y otro no deseable. Ambos estilos correlacionaron negativamente con la mayoría de las dimensiones del CFP y positivamente con las del ECO, pero el no deseable obtuvo coeficientes más débiles. El impacto del factor organizacional y del de relaciones (ambos con coeficiente negativo) y las manifestaciones de salud (coeficiente positivo), junto con el apoyo, predijeron el liderazgo deseable. El apoyo (coeficiente positivo) y el control (coeficiente negativo), predijeron el liderazgo no deseable.
\end{abstract}

Palabras clave: Liderazgo, clima organizacional, riesgo psicosocial, bienestar.

Investigación realizada con fondos propios por el Grupo de Investigación en Perdurabilidad Empresarial de la Universidad del Rosario (Colombia), línea de investigación de liderazgo.

* PHD en Psicología, Universidad Autónoma de Madrid, Madrid, España, 2005. Magister Evaluación de la Educación, Universidad Santo Tomás de Colombia, Bogotá, Colombia, 2000. Psicóloga, Universidad Santo Tomás, Bogotá, Colombia, 1990. Profesora Titular, Facultad de Administración, Universidad del Rosario, Bogotá, Colombia. Miembro del Grupo en investigación GIPE. Correo electrónico: francoise.contreras@urosario.edu.co

*** PHD en Psicología, Universidad Autónoma de Madrid, Madrid, España, 1997. Psicólogo, Universidad Autónoma de Madrid, Madrid, España, 1991. Profesor Asociado, Facultad de Administración, Universidad del Rosario, Bogotá, Colombia. Miembro del Grupo en investigación GIPE. Correo electrónico: fernando.juarez@urosario.edu.co

Magister en Administración de Empresas, Universidad de los Andes, Bogotá, Colombia, 2006. Abogado, Universidad del Rosario, Bogotá, Colombia, 1997. Médico y Cirujano, Pontificia Universidad Javeriana, Bogotá, Colombia, 1992. Profesor Principal, Facultad de Administración, Universidad del Rosario, Bogotá, Colombia. Miembro del Grupo en investigación GIPE. Correo electrónico: david.barbosa@urosario.edu.co

PHD en Psicología Clínica y de la Salud, Universidad de Granada, Granada, España, 2005. Magister en Educación y Prevención del SIDA, Universidad Complutense de Madrid, Madrid, España, 2002. Psicóloga, Pontificia Universidad Javeriana, Cali, Colombia, 1997. Directora de la Facultad de Psicología de la Universidad Pontificia Bolivariana, Bucaramanga, Colombia. anafernanda. uribe@upbbga.edu.co 


\title{
LEADERSHIP STYLES, PSYCHOSOCIAL RISK AND ORGANIZATIONAL CLIMATE IN A GROUP OF COMPANIES
}

\begin{abstract}
This research analyzed the relationships among psychosocial risks, organizational climate and leadership style in Colombian companies from social and health services sector. Four hundred participants answered the Organizational Climate Scale (OCS), the questionnaire of Psychosocial Factors at Work (PFW) and Pitcher Adjective Test [PAT $\neg$ ]. Correlation coefficients and linear regressions were obtained. In general, the OCS dimensions were negatively correlated to PFW. Two leadership styles were obtained from PAT, one desirable and the other undesirable. Both styles correlated negatively with most of PFW dimensions and positively with OCS dimensions, but the one undesirable obtained a weaker coefficient. The impact of organizational factor and relationships (both with negative coefficient) and health manifestations (positive coefficient), together with support, predicted the desirable leadership. While support (positive coefficient) and control (negative coefficient), predicted the undesirable leadership.
\end{abstract}

Key words: Leadership, organizational climate, psychosocial risk, welfare.

\section{ESTILOSDE LIDERANÇA, RISCO PSICOSSOCIAL E CLIMA ORGANIZACIONAL EM UM GRUPO DE FIRMAS}

\begin{abstract}
Resumo
Esteestudo analisou a relação entre os riscos psicossociais, o clima organizacional e o estilo de liderança em empresas colombianas do sector dos serviços sociais e de saúde. Quatrocentos participantes responderam a Escala de Clima Organizacional (ECO), o Questionário de Fatores Psicossociais no Trabalho (QFP) e o Teste de Adjetivos de Pitcher (Pitcher Test Adjectives - PAT). Obtiveram-secoeficientes decorrelaçãoe deregressãolinear. Emgeral, acorrelaçãoentreasdimensões daECO eas do QFP foram negativas. NoPAT, apresentaram-se dois estilos de liderança: um desejávele outro indesejável. A correlação de ambos os estilos com a maioria das dimensões do PCP foi negativa, e positiva com as do ECO. O não desejável obtive coeficientes mais fracos. O efeito dos fatores organizacionais e de relaciones (ambos com coeficiente negativo) e as manifestações de saúde (coeficiente positivo), com o apoio, predisseram uma liderança desejável. O apoio (coeficiente positivo) e o controle (coeficiente negativo) predisserama liderança não desejável.

Palavras chave: Liderança, clima organizacional, de risco psicossocial, bem-estar.
\end{abstract}

Contresas, F., Juárez, F., Barbosa, D. \& Uribe, A. (2010) Estilos de liderazgo, riesgo psicosocial y clima organizacional en un grupo de empresas colombianas. En: Revista de la Facultad de Ciencias Económicas de la Universidad Militar Nueva Granada. rev.fac.cienc.econ, XVIII (2)

JEL: M30, M29, M44.

\section{Introducción}

El liderazgo juega un rol vital en las organizaciones, al proveer dirección y facilitar procesos para el logro de metas y objetivos organizacionales (Zaccaro \& Klimoski, 2001) ejerciendo un efecto importante en la implementación de estándares de comportamiento en las organizaciones (Grojean, Resick, Dickson \& Smith, 2004), normas sociales y expectativas de comportamiento (Srivastav, 2005), al mediar las percepciones de los métodos y procesos organizacionales (Peiró, 1995). La influencia de los 
líderes se produce a través del mantenimiento de normas, de la calidad de las relaciones interpersonales y al convertirse en modelo para los colaboradores (Grojean, et al., 2004). De este modo, el líder modula la forma en que los individuos perciben la organización y así, en la percepción del clima y de los aspectos psicosociales de la misma.

En general, existen dos grandes estilos de liderazgo, el estilo centrado en las personas y el estilo centrado en el cumplimiento de objetivos organizacionales. El primero tiene en cuenta las características situacionales, persuadiendo a los colaboradores para el logro de metas comunes y generando compromiso y satisfacción en los equipos, mientras que el segundo puede descuidar aspectos como la motivación, el clima organizacional y otras características relacionadas con la satisfacción y bienestar del trabajador. Esto último, incide en la calidad del desempeño (Conger, Kanungo \& Menon, 2000; Denison, 1990; Kleinman, 2004; Jung \& Avolio, 2000; Patterson, et al, 2005; Patterson, Warr \& West, 2004). Los líderes centrados en la persona promueven la productividad de la empresa, al mejorar el desempeño de los trabajadores a través de un buen clima organizacional (Carr, Schmidt, Ford \& DeShon, 2003; Singh, 1998). Por su parte, los líderes centrados en los objetivos organizacionales ejercen más control sobre la tarea imponiendo altos niveles de coerción y exigencia; esto afecta el clima organizacional, al deteriorar las relaciones al interior de la organización (Bloch \& Whiteley, 2003).

De acuerdo con lo anterior, existe amplio consenso respecto al efecto del liderazgo en los estados emocionales, las creencias, las conductas y el compromiso de los trabajadores, el bienestar psicológico, el desempeño laboral y el clima organizacional (Carr, et al., 2003; Contreras, Barbosa, Juárez, Uribe \&, Mejía, 2009; Waldman et al., 2006). Este último, el clima organizacional consiste en las percepciones compartidas de los atributos de una organización y sus subsistemas (Reichers \& Schneider, 1990; Srivastav, 2005) y en la influencia de dicha percepción sobre la relación de la organización con sus miembros y los grupos de interés (Pareek, 2004).
Así pues, el clima organizacional está relacionado con las características del líder, el cual ejerce un fuerte efecto sobre el mismo. El clima, a su vez, incide en la motivación (Srivastav, 2006), en el desempeño laboral y la salud de los trabajadores (Escobar, Dávila, Mulett \& Uribe, 2007), así como en la eficiencia, satisfacción percibida, afecto al trabajo y con el nivel de ausentismo laboral (Toro, 1998). De esta manera el clima se relaciona con la manera en que se perciben y se sienten los efectos de los factores psicosociales de riesgo en la organización.

Esto se hace evidente en cuanto que los climas organizacionales funcionales reducen el estrés experimentado en la actividad laboral y promueven la salud física y mental de los empleados (Srivastav, 2006), mientras que los climas disfuncionales se caracterizan por la percepción de la existencia de riesgos psicosociales y de sus efectos sobre el bienestar, la satisfacción de las personas y la productividad. La presencia de factores de riesgo psicosociales, tanto laborales como extralaborales, deterioran la salud de los trabajadores, dando lugar a problemas asociados al estrés laboral (Aranda, Pando, Salazar, Torres, Aldrede \& Perez, 2004) y a la accidentalidad en el trabajo (Rentería, Fernández, Tenjo \& Uribe, 2009). Además, el incremento en el apoyo, la recompensa y el confort físico, características específicas del clima, ocasionan una menor percepción de riesgo psicosocial, mientras que el control y la presión, otras características del clima, incrementan dicha percepción (Contreras, et al., 2009).

La relación entre el riesgo psicosocial, el clima laboral y el estilo de liderazgo, es compleja, ya que el clima puede cambiar tanto a través de los subordinados como de los administradores que influyen en políticas y procedimientos organizacionales (Adamek, 2007; King, 2007). Igualmente, los factores de riesgo psicosocial deterioran las relaciones, incidiendo sobre el clima organizacional; de hecho, la existencia del factor de riesgo asociado a las relaciones es frecuente y de gran impacto en las organizaciones (Contreras, et al., 2009). De esta manera, intervenir en los factores de riesgo, mejora el desempeño del individuo y el cumplimiento de los objetivos estratégicos (Uribe, 2007), aspectos que 
preocupan especialmente al líder centrado en los objetivos. A su vez, la preocupación por el bienestar de los trabajadores, caracteriza a las empresas socialmente responsables, al centrarse en el factor humano como propósito y función de las mismas (Contreras, et al., 2009), lo que caracteriza al líder centrado en la persona.

De esta manera, los comportamientos del líder influyen en la presencia o no de los riesgos psicosociales. Así, la claridad en los objetivos y en la toma de decisiones, al igual que la calidad en la comunicación, constituyen factores protectores de la salud que promueven la satisfacción y el bienestar, reduciendo los riesgos psicosociales. Por el contrario, altas demandas, bajo control percibido y altos esfuerzos unidos a bajas recompensas, constituyen factores de riesgo para la salud (Stansfeld, Bosman, Hemingway \& Marmot, 1998), al igual que la carencia de roles claros, falta de autonomía (Niedhammer, Bugel, Goldberg, Leclerc \& Gueguen, 1998), escaso apoyo y la hostilidad (Wilkinson \& Wagner, 1993), todos ellos factores que afectan el bienestar de los individuos en las organizaciones.

Todo lo anterior, pone de manifiesto la estrecha y compleja relación entre el estilo de liderazgo, la percepción de riesgo psicosocial y el clima organizacional. Debido a todo esto, y ya que el estilo de liderazgo produce un impacto en el clima organizacional y en la existencia de riesgos psicosociales, sería posible determinar qué estilo de liderazgo predomina, a partir de la existencia de determinados riesgo psicosociales y de clima organizacional. No obstante, el efecto del liderazgo sobre los riesgos psicosociales parece depender de factores culturales, en ciertos contextos, un estilo de liderazgo no deseable (volátil, impredecible, difícil, inflexible, testarudo y extremista), hace que los trabajadores perciban menores riesgos psicosociales, mientras que el estilo deseable (orientado hacia las personas, racional y metódico, amistoso y cortés) predice que se perciban mayores riesgos, lo que puede ser explicado por la existencia de patrones culturales no suficientemente conocidos (Contreras, et al., 2009).

Con base a lo anterior, el propósito de este estudio es observar las relaciones existentes entre el liderazgo, la percepción de riesgos psicosociales y el clima organizacional, en un grupo de empresas colombianas pertenecientes al sector de servicios sociales y salud, estableciendo si se puede predecir el estilo de liderazgo a partir del clima laboral y los factores de riesgo psicosocial existentes.

\section{Método}

\subsection{Participantes}

En el estudio participaron 400 trabajadores $(63,8 \%$ mujeres y 36,2\% hombres) de Entidades Promotoras de Salud (EPS), pertenecientes al sector de servicios sociales y salud, en cuatro ciudades importantes del país, como son Barranquilla, Bogota, Cali y Bucaramanga. Los trabajadores que hicieron parte del estudio tenían una edad promedio de 30.2 años (desviación estándar de 9.73). Su antigüedad promedio en la empresa era de 43.4 meses (desviación estándar de 43.5), distribuyéndose entre administrativos ( $\mathrm{F}=162,40.5 \%)$, operarios ( $\mathrm{F}=119,29.8 \%)$, ejecutivos/directivos ( $\mathrm{F}=86,21.5 \%)$ y técnicos $(\mathrm{F}=33$, 8.3\%). Su nivel de escolaridad era principalmente de técnicos $(\mathrm{F}=175,43.8 \%)$ y profesionales ( $\mathrm{F}=139,34.8 \%)$ y algo más de la mitad pertenecían al estrato socioeconómico tres $(\mathrm{F}=203,50,8 \%)$ siguiéndole el estrato dos ( $\mathrm{F}=87,21,8 \%)$.

\subsection{Instrumentos (ESTA)}

Cuestionario de Factores Psicosociales en el trabajo [CFP]. Este instrumento, diseñado por Espinosa y Romero (2002), evalúa tres aspectos, 1) presencia de los factores de riesgo psicosocial, 2) el impacto que se percibe por la presencia de dichos riesgos y 3) las manifestaciones asociadas a los mismos (frecuencia de síntomas psicológicos y biológicos asociados con el estrés ocupacional y las enfermedades profesionales). El instrumento presentó un alto nivel de confiabilidad en los tres aspectos mencionados (alpha de Cronbach de 0.84, 0.90 y 0.86 respectivamente).

Los factores de riesgo evaluados por el instrumento son: a) Labores y condiciones de su realización (apremio de tiempo, complejidad de la tarea, sobrecarga, monotonía, autonomía, y responsabilidad, etc.), b) Organización del trabajo y gestión empre- 
sarial (pausas durante la jornada, rotación del personal, cambios organizacionales, estilos de mando, niveles de participación, políticas de desarrollo del talento humano, etc.), c) Relaciones interpersonales (comunicación, resolución de conflictos entre grupos, valores éticos, compromiso, confianza, respeto, tolerancia, etc.), y d) Factores extralaborales que inciden en el trabajo (relaciones de pareja, familiares y sociales).

Test de adjetivos de Pitcher [PAT]. Instrumento desarrollado por Pitcher (1997), compuesto por 60 adjetivos, a través de los cuales los individuos evalúan a su propio jefe. La autora del instrumento evaluó tres estilos de liderazgo los cuales denominó artesanos (razonables, confiables sensibles), tecnócratas (líderes analíticos, brillantes y fríos) y artistas (líderes generosos, cálidos y emocionales) (Pitcher, 1999). Mediante un análisis de correspondencias realizado con los adjetivos asignados a cada líder, se obtuvieron dos dimensiones que explicaban el $69 \%$ de la varianza. Por otra parte, mediante análisis factorial realizado sobre las características de un grupo de líderes, se obtuvo un agrupamiento en tres dimensiones (los tres estilos de líderes) explicando el $75,1 \%$ de la varianza. Así, la correspondencia entre los adjetivos dio lugar a dos dimensiones, mientras que el análisis factorial permitió establecer la validez criterial del instrumento. No existen datos relativos a la confiabilidad del test.

Escala de clima organizacional [ECO]. Escala multidimensional desarrollada por Fernandes (2008) cuyo objetivo es evaluar la percepción de los trabajadores sobre varias dimensiones del clima organizacional. Está compuesta por 63 afirmaciones y cinco alternativas de respuesta, que agrupa cinco factores: 1) Apoyo del jefe y de la organización (soporte afectivo, estructural y operacional), 2) Recompensa (diversas formas en las que la empresa premia la calidad, la productividad y el esfuerzo de los trabajadores), 3) Confort físico (ambiente físico y seguridad, proporcionada por la empresa), 4) Control/presión (ejercido por la empresa o los supervisores sobre el comportamiento y desempeño de los empleados) y 5) Cohesión entre colegas (unión, vínculos de colaboración entre compañeros de trabajo). La escala ha demostrado ser altamente confia- ble, obteniendo coeficientes alpha de Cronbach de 0.92 para la dimensión de Apoyo, de 0.88 para la dimensión de Recompensa, de 0.86 para la dimensión de Confort físico, de 0.78 para la dimensión de Control y de 0.78 para la dimensión de Cohesión.

\subsection{Procedimiento}

Una vez obtenido el permiso de cada una de las EPS, en las cuatro ciudades que hicieron parte del estudio, así como el consentimiento informado de los participantes, se procedió a la aplicación colectiva por grupos, de los instrumentos en cada institución. Se informó a los participantes que su inclusión en el estudio era voluntaria, anónima y que no obtendrían incentivos económicos por su participación.

\section{Resultados}

Inicialmente se estudió la relación entre la presencia e impacto de los riesgos psicosociales evaluados y la percepción de clima organizacional que tenían los trabajadores. Como puede observarse en la Tabla 1, se evidencia que a mayor presencia de riesgos psicosociales asociados con las labores (factor laboral), esto es, condiciones para su realización, apremio de tiempo, sobrecarga y monotonía, entre otras, se percibe un clima laboral con mayor control y menor apoyo y recompensa. Así mismo, se observan relaciones inversas entre el impacto percibido en este factor de riesgo sobre todas las variables del clima organizacional estudiadas, exceptuando el control, en donde la correlación no fue significativa. Con respecto al factor organizacional, que tiene que ver básicamente con la gestión empresarial, como pausas durante la jornada, rotación del personal, niveles de participación, políticas de desarrollo del talento humano, entre otras, se observa una correlación inversa y significativa entre la percepción de riesgo en este factor y todas las variables del clima organizacional, exceptuando nuevamente el control. El impacto que se percibe con respecto a este factor, correlaciona también de manera inversa con las variables de clima, incluyendo el control.

El factor relacional o de relaciones interpersonales, que incluye la comunicación, resolución de conflictos, valores éticos, compromiso, confianza, respeto, 
tolerancia, entre otras, correlacionó de manera inversa y significativa con apoyo, recompensa y cohesión. A nivel de impacto, este factor al igual que el organizacional se asoció inversamente con todas las variables de clima, estos es, apoyo, recompensa, confort, control y cohesión. Finalmente los factores de riesgo psicosocial asociados con aspectos extralaborales y que pueden afectar el trabajo, tales como las relaciones de pareja, familiares y sociales, correlacionaron de manera inversa con apoyo y re- compensa. De manera similar el nivel de impacto en éste factor se relaciona de manera inversa con el apoyo, la recompensa y el control.

Con respecto a las manifestaciones sobre la salud (frecuencia de síntomas psicológicos y biológicos) asociadas con la presencia de los riesgos psicosociales, se encontró una relación inversa con apoyo, recompensa, confort y cohesión.

Tabla 1. Relación entre la presencia e impacto de los factores de riesgo psicosocial y las dimensiones del clima organizacional percibido por el grupo de trabajadores ${ }^{1}$

\begin{tabular}{|c|c|c|c|c|c|c|c|c|c|c|}
\hline \multirow[t]{3}{*}{ Riesgo Psicosocial } & \multicolumn{10}{|c|}{ Clima Organizacional } \\
\hline & \multicolumn{2}{|c|}{ Apoyo } & \multicolumn{2}{|c|}{ Recompensa } & \multicolumn{2}{|c|}{ Confort } & \multicolumn{2}{|c|}{ Control } & \multicolumn{2}{|c|}{ Cohesión } \\
\hline & -.22 & ** & -.26 & $\star \star *$ & .01 & & .10 & * & -.05 & \\
\hline Factor organizacional & -.30 & ** & -.35 & ** & -.15 & ** & -.05 & & -.20 & ** \\
\hline Factor relacional & -.23 & ** & -.24 & $\star \star$ & .02 & & .07 & & -.12 & * \\
\hline Factor extralaboral & -.20 & $* \star$ & -.22 & $* *$ & .04 & & .09 & & -.02 & \\
\hline Impacto factor laboral & -.26 & ** & -.21 & ** & -.12 & * & -.09 & & -.21 & ** \\
\hline Impacto factor organizacional & -.63 & ** & -.56 & ** & -.45 & ** & -.20 & ** & -.46 & ** \\
\hline Impacto factor relacional & -.58 & ** & -.36 & ** & -.39 & ** & -.17 & ** & -.51 & ** \\
\hline Impacto factor extralaboral & -.13 & * & -.14 & $\star \star$ & -.07 & & -.11 & * & -.07 & \\
\hline Manifestaciones sobre la salud & -.18 & $\star \star$ & -.19 & ** & -.19 & ** & -.07 & & -.19 & ** \\
\hline
\end{tabular}

${ }^{\star} \mathrm{p} \leq, 05 ;{ }^{* *} \mathrm{p} \leq, 01$

A manera de resumen, en la Tabla 1 puede notarse que la percepción de apoyo y recompensa, características deseables del clima organizacional, se asocian negativamente con todos los factores de riesgo psicosocial evaluados, tanto en presencia como en impacto. En el caso del confort, otra característica que se asume favorable para el clima organizacional, se encuentra asociado negativamente con el factor organizacional, tanto en presencia como en impacto, así como con el impacto del factor laboral y relacional. Por otra parte, el control, que se presume afecta el clima organizacional, se encontró asociado positivamente con el factor laboral, pero negativamente con el impacto de los factores organizacional, relacional y extralaboral. En este senti- do, es posible que a mayor control las personas perciban menor impacto de dichos riesgos psicosociales, pero perciben mayor exposición a los mismos, exceptuando al factor organizacional. Finalmente, la cohesión correlacionó de manera negativa con la presencia y el impacto en los factores de riesgo organizacional y de relaciones; con el factor laboral correlacionó únicamente en el impacto.

El liderazgo fue evaluado a partir de las dos categorías resultantes en un estudio previo (Contreras, et al., 2009) en el que se utilizo el test de adjetivos de Pitcher (PAT), compuesto por 64 adjetivos. Estas categoría se denominaron Líder 1 (deseable) y Líder 2 (no deseable) y los adjetivos más representativos

1 Tabla de la investigación. 
(y su saturación correspondiente en la categoría de Líder 1 (deseable) fueron: Humano (.339), Equilibrado (.327), Orientado hacia las personas (.373), Reflexivo (.325), Colaborador (.334), Honesto (.430), Franco, (.392), Brillante (.310), Racional (.371), Cálido (.321), Digno de confianza (.455), Detallista (.386), Generoso (.412), Realista, (.437), Abierto (.316), Analítico (.361), Emprendedor (.386), Firme (.331), Cortés (.371) y Razonable (.433). Los adjetivos más representativos (y su saturación correspondiente en la categoría) en la categoría de Líder 2 (no deseable) fueron: Impredecible (.298), Difícil (.401), Inflexible (.368), Rígido (.316), Extremista (.360), Fastidioso (.301) y Testarudo (.357). Ninguno de los adjetivos indicados obtuvo saturaciones altas simultáneamente en las dos categorías. Estas dos categorías se correlacionaron con las variables de clima organizacional y de riesgo psicosocial. En la Tabla 2, pueden observarse dichas correlaciones.

Tabla 2. Correlaciones entre los factores de riesgo psicosocial, las dimensiones del clima organizacional y las categorías de liderazgo²

\begin{tabular}{|c|c|c|c|c|}
\hline \multirow{2}{*}{$\begin{array}{l}\text { Dimensiones } \\
\text { RIESGO PSICOSOCIAL }\end{array}$} & \multicolumn{2}{|c|}{$\begin{array}{c}\text { Líder } 1 \\
\text { (deseable) }\end{array}$} & \multicolumn{2}{|c|}{$\begin{array}{c}\text { Líder } 2 \\
\text { (no deseable) }\end{array}$} \\
\hline & & & & \\
\hline Factor Laboral & -.220 & $\star *$ & -.102 & * \\
\hline Factor Organizacional & -.189 & $\star \star$ &,- 085 & \\
\hline Factor Relacional & -.192 & $\star \star$ &,- 067 & \\
\hline Factor Extralaboral & -.196 & ** & -.108 & * \\
\hline Impacto Factor Laboral & -.196 & $\star \star$ &,- 013 & \\
\hline Impacto Factor Organizacional & -.406 & $\star \star$ & -.152 & $\star \star$ \\
\hline Impacto Factor Relacional & -.332 & $\star \star$ & -.146 & $\star \star$ \\
\hline Impacto Factor Extralaboral &, 010 & &, 007 & \\
\hline Manifestaciones sobre la salud & 029 & & -.108 & * \\
\hline \multicolumn{5}{|l|}{ CLIMA ORGANIZACIONAL } \\
\hline Apoyo & .468 & ** & .242 & $\star \star$ \\
\hline Recompensa & .353 & $\star *$ & .157 & $* \star$ \\
\hline Confort & .220 & $\star \star$ & .148 & $\star *$ \\
\hline Control & .103 & * &,- 089 & \\
\hline Cohesión & .225 & $\star \star$ & .191 & ** \\
\hline
\end{tabular}

${ }^{*} \mathrm{p} \leq, 05 ;{ }^{* *} \mathrm{p} \leq, 01$

2 Tabla de la investigación.
Como puede observarse en la Tabla 2, la categoría de Líder 1 (deseable) se encuentra asociada negativamente con todas las dimensiones de riesgo psicosocial, a excepción del impacto de la dimensión extralaboral y las manifestaciones que tienen los factores sobre la salud, las cuales no resultan significativas, siendo las correlaciones más elevadas las obtenidas con los factores de impacto organizacional y relacional. Así mismo, se observa que la categoría de Líder 1, se encuentra asociado de forma positiva con las dimensiones del clima organizacional, en especial con las dimensiones apoyo y recompensa las cuales presentan una asociación moderada (,468 y ,353 respectivamente); las otras tres dimensiones del clima presentaron una correlación baja con la categoría de Líder 1 .

Por otra parte, la categoría de Líder 2 (no deseable) se encuentra asociado, al igual que el Líder 1 , de forma negativa, aunque con coeficientes más débiles, con las dimensiones de riesgo psicosocial, si bien solamente resultaron significativas las correlaciones con los factores laboral, extralaboral y de impacto de los factores organizacional y relacional. A diferencia de la categoría de Líder 1, el Líder 2 se encuentra asociado de forma significativamente negativa, aunque con un coeficiente reducido, con las manifestaciones sobre la salud. Por otra parte, de manera similar a la categoría Líder 1 , la categoría de Líder 2 se encuentra asociada positivamente con las dimensiones apoyo, recompensa, confort y cohesión, aunque en todos los casos la correlación es menor en el Líder 2 que en el Líder 1 . Otra diferencia es que la categoría de Líder 2 no muestra una asociación significativa con la dimensión control, mientras que la categoría de Líder 1 muestra una asociación positiva, si bien en ambos casos los coeficientes de correlación son reducidos.

Finalmente, para evaluar si es posible predecir la existencia de un tipo de líder u otro, a partir de su efecto en el ambiente, se realizó una regresión lineal múltiple utilizando las dimensiones de riesgo psicosocial y de clima organizacional como variables independientes y la categoría de líder ( 1 o 2) como variable dependiente; en la Tabla 3 se muestran los resultados de la regresión. 
Tabla 3. Coeficientes de la regresión lineal múltiple sobre los dos tipos de líderes ${ }^{3}$

\begin{tabular}{|l|r|r|r|r|}
\hline \multicolumn{1}{|c|}{ Dimensiones } & \multicolumn{2}{c|}{ LíDER 1 } & \multicolumn{2}{c|}{ LíDER 2 } \\
\hline FACTORES DE RIESGO & \multicolumn{2}{c|}{ B } & \multicolumn{2}{c|}{ B } \\
\hline PSICOSOCIAL & & & & \\
\hline Factor Laboral & & & & \\
\hline Factor Organizacional &,- 294 & & 2,201 & \\
\hline Factor Relacional &,- 068 & &,- 135 & \\
\hline Factor Extralaboral &, 323 & &, 516 & \\
\hline Impacto Factor Laboral &,- 243 & &,- 409 & \\
\hline Impacto Factor Organizacional &,- 189 & &, 332 & \\
\hline Impacto Factor Relacional &,- 500 & $*$ &,- 017 & \\
Impacto Factor Extralaboral &, 267 & &,- 227 & \\
Manifestaciones sobre la salud &, 452 & $*$ &,- 372 & \\
CLIMA ORGANIZACIONAL & & & & \\
Apoyo & 1,450 & $* *$ &, 800 & $*$ \\
Recompensa &, 282 & &,- 044 & \\
Confort &,- 259 & &, 147 & \\
Control &,- 069 & & $-1,030$ & $*$ \\
Cohesión &,- 499 & &, 469 & \\
\hline
\end{tabular}

${ }^{*} p \leq, 05 ;{ }^{*} p \leq, 01$

La regresión sobre la categoría de Lider1 (deseable) resultó significativa $\left(F=10,671 p=.000, R^{2}\right.$ $=.28$ ), así como las dimensiones de los factores de riesgo de impacto del factor organizacional, impacto del factor de relaciones, ambos con coeficientes negativos, y las manifestaciones de salud, esta con coeficiente positivo, tal como se observa en la Tabla 3; a su vez, resulta significativa la dimensión de apoyo del clima organizacional, con coeficiente positivo. Igualmente, la regresión sobre la categoría de Líder 2 (no deseable) resulta significativa ( $\mathrm{F}=3,513$ $\left.p=.000, R^{2}=.11\right)$, pero, tal y como se observa en la misma tabla, no resultó significativa ninguna de las dimensiones de los factores de riesgo, aunque sí las dimensiones de apoyo y control del clima organizacional, la primera con coeficiente positivo y la segunda con coeficiente negativo.

\section{Discusión}

Una de las características que se han analizado en nuestro estudio, es la asociación entre clima laboral y los factores de riesgo psicosocial, ya que o bien el clima laboral se ha considerado un precursor de los factores de riesgo psicosocial (Coyle, Sleeman \& Adams, 1995) o, por el contrario, el impacto de ciertos factores de riesgo hace que se perciba el clima laboral de manera adversa (Martín, Luceño, Jaén \& Rubio, 2007); en este sentido, los resultados confirman dicha relación inversa, ya que muestran que, en general, a mejor clima laboral menor percepción de riesgo psicosocial y a mayor percepción de riesgo psicosocial peor clima laboral. Solamente existió una correlación significativa positiva, la existente entre la dimensión de control y el factor laboral de riesgo, si bien dicha correlación es relativamente reducida (ver Tabla 1). Estos resultados son coincidentes, también, con lo expresado en otros estudios, donde se señala que el clima organizacional funcional reduce el estrés y favorece el bienestar (Srivastav, 2006), mientras que los climas disfuncionales conducen a la existencia de riesgos (Peiró, 1999), a una mayor percepción de riesgo psicosocial, problemas de salud (Aranda, et al., 2004) y a un deterioro del clima laboral (Contreras, et al., 2009), produciéndose conductas como el moobing (Meseguer, Soler, García-Izquierdo, Sáez \& Sánchez, 2007).

La dimensión de control del clima laboral, ofrece resultados contrarios a lo que intuitivamente se podría esperar, ya que un aumento en las condiciones de control se asocia con una reducción del impacto de todos los factores de riesgo, exceptuando el factor laboral, si bien se asocia positivamente con la frecuencia de presencia de dicho factor. Esto puede deberse al efecto positivo que tiene el mantenimiento de las normas por el líder (Grojean, et al., 2004). Otra explicación tendría que ver con los patrones culturales, los cuales pueden hacer que la percepción de control reduzca el impacto del riesgo en los individuos (Contreras, et al., 2009), generando mayor resistencia a los factores de riesgo psicosocial.

3 Datos de la investigacion. 
Los dos tipos de líderes obtenidos (deseable y no deseable) muestran un patrón de asociación similar con los factores de riesgo psicosocial y dimensiones de clima laboral, es decir, asociaciones negativas significativas con los factores de riesgo psicosocial y asociaciones positivas significativas con las dimensiones del clima laboral. La diferencia reside en mayores coeficientes, o una asociación más fuerte, para el líder deseable (Líder 1) en contraposición al líder no deseable (Líder 2), así como en algunas correlaciones significativas en uno de los líderes y no en el otro, como la obtenida para las manifestaciones de salud o la dimensión de control. Estos dos estilos de liderazgo parecen coincidentes con las categorías de líder centrado en las personas y líder centrado en los resultados (Conger, et al., 2000; Denison, 1990; Kleinman, 2004; Jung \& Avolio, 2000; Patterson, et al., 2005; Patterson, et al., 2004). Así, el líder no deseable tiene un efecto más reducido que el líder deseable, en la amortiguación de los factores de riesgo psicosocial y en el mejoramiento de las dimensiones del clima laboral. Esto, también es coincidente con lo propuesto por algunos autores cuando señalan que el líder centrado en las personas, en nuestro caso deseable, ejerce un efecto positivo en el clima organizacional (Carr, et al., 2003; Singh, 1998), mientras que el líder centrado en los objetivos y resultados, en nuestro caso el líder no deseable, produce un deterioro del clima organizacional (Bloch \& Whiteley, 2003).

Sin embargo, en esta ocasión, el efecto en el clima organizacional y los factores de riesgo psicosocial, que se puede atribuir al líder no deseable, no se produce por un incremento de dichos factores de riesgo y un deterioro del mencionado clima organizacional, ya que este líder se asocia negativamente con los factores de riesgo psicosocial y positivamente con las dimensiones de clima laboral, sino por la falta de potenciación tanto en la amortiguación de los factores de riesgo como en el incremento de las dimensiones positivas del clima organizacional. Se podría decir que un líder con las características del líder no deseable, no va a ejercer la suficiente influencia como para realizar una acción preventiva y protectora. Es también en este sentido, que se podría entender el efecto obtenido en la dimensión de control, la cual se asocia positivamente con el líder deseable negativamente con el líder no deseable, ya que el control podría imponer una serie de normas y comportamientos que se percibieran por los individuos como tendentes a proporcionarles seguridad. Esto concuerda con el hecho de que en la interpretación del comportamiento del líder, que se puede hacer por los subordinados, se deben tener en cuenta los poderosos efectos culturales, los cuales moldean dicha conducta (Castro \& Lupano, 2005) y también con el hecho de que el líder puede favorecer cambios organizacionales, los cuales están influenciados por los factores de riesgo psicosocial (García, Rubio \& Bravo, 2007), lo que pone de manifiesto que el líder puede reducir el impacto de estos factores. Un líder ejerce un efecto de contención de diferentes tipos de problemas (Medina, 2004).

Finalmente, ya que se daban estas relaciones entre el estilo de liderazgo y la percepción de los factores de riesgo y del clima organizacional, parecía posible predecir, a partir de las dimensiones de riesgo psicosocial y de clima laboral, el tipo de líder que estaba presente en la organización. Esto está en concordancia con lo indicado acerca de que el líder origina efectos en la institución (Grojean, et al., 2004) y de que un cierto tipo de líder también tiene impacto en el clima laboral y en los factores de riesgo (Conger, et al., 2000; Denison, 1990; Kleinman, 2004; Jung \& Avolio, 2000; Patterson, et al, 2005; Patterson, et al., 2004). De acuerdo con esto, observando dicho clima laboral y los factores de riesgo existentes, sería posible establecer el tipo de líder presente.

De acuerdo a los resultados (Tabla 3), las dimensiones de impacto del factor organizacional, impacto del factor relacional (ambos con coeficientes negativos) y las manifestaciones sobre la salud (coeficiente positivo), predicen de manera inversa la existencia del líder deseable; a su vez, en esta predicción también ejerce una influencia directa (coeficiente positivo) la dimensión de apoyo, del clima organizacional. Así, la predicción es compleja, ya que el mayor apoyo, el reducido impacto de los factores de riesgo anteriores y una menor salud, son característicos de aquellas organizaciones con un líder deseable. Es decir, no todas las dimensiones evolucionan en sentido positivo ante la presencia de este tipo de líderes. 
Por otra parte, únicamente las dimensiones de apoyo y control, esta última en relación inversa, guardan relación con el líder no deseable, permitiendo predecir la existencia de este tipo de líderes. Es decir, el mayor apoyo y el menor control permiten afirmar la existencia de un líder no deseable.

De esta manera, existen más dimensiones en la predicción del líder deseable que del no deseable, pero, por otra parte, dichas dimensiones no evolucionan de acuerdo a lo que intuitivamente podríamos pensar a partir de las características de cada uno de los líderes. Sin embargo, la explicación aportada por ambas regresiones es baja, siendo ligeramente mayor la explicación de la regresión sobre líder deseable (28\%) que la de líder no deseable (11\%), lo que indica que la predicción realizada a partir de estas variables resulta muy débil.

De esta manera, aunque en diversas ocasiones se pone de manifiesto los efectos asociados a la presencia de un estilo u otro de liderazgo, cuando se intenta predecir la existencia de alguno de dichos estilos a partir de las variables supuestamente afectadas por los mismos, como ha ocurrido en nuestro estudio, esto se verifica en un grado muy reducido, ofreciendo además resultados que no confirman que esos efectos se producen por la existencia de esos líderes.

De acuerdo con lo anterior, si bien es cierto, tal como se ha obtenido en este estudio que existen relaciones entre los estilos de liderazgo, la percepción de factores de riesgo psicosocial y el clima organizacional, la predicción de la existencia de un cierto estilo de liderazgo en una organización, realizada a partir de las variables de riesgo psicosocial y de clima laboral resulta débil y no confirma los efectos descritos para cada estilo de liderazgo. Esto hace necesario profundizar tanto en estas relaciones establecidas, como en la existencia de otras posibles variables que puedan estar mediando en las predicciones realizadas, antes de elaborar un modelo de influencia.

\section{Referencias}

Adamek, M (2007). Elements of Leadership Development: What Contributes to Effective Leadership? En: Music Therapy Perspectives, 25: 121-124.
Aranda, C, Pando, M, Salazar, J, Torres, T, Aldrede, M. \& Pérez, M. (2004). Factores psicosociales laborales y síndrome del burnout. En: Médicos del primer nivel de atención. Investigación en Salud, 6: 28-34.

Bloch, S. \& Whiteley, P. (2003). Complete Leadership: A practical guide for developing your leadership talents. London: Pearson.

Carr, J, Schmidt, A, Ford, J. \& De Shon, R. (2003). Climate perceptions matter: A meta-analytic path analysis relating molar climate, cognitive and affective states, and individual level work outcome. En: Journal of Applied Psychology, 88: 605619.

Castro, A. \& Lupano, I. (2005). Diferencias individuales en las teorías implícitas del liderazgo y la cultura organizacional percibida. Boletín de Psicología, 85, 89-109.

Conger J, Kanungo, R. \& Menon, S. (2000). Charismatic leadership and follower effects. En: Journal of Organizational Behavior, 21: 747-767.

Contreras, F, Barbosa, D, Juárez, F, Uribe, A, \& Mejía, C. (2009). Estilos de liderazgo, clima organizacional y riesgos psicosociales en entidades del sector salud, como criterios de Responsabilidad Social Empresarial. Estudio comparativo en cuatro ciudades colombianas. En: Revista Acta Colombiana de Psicología. 12: 13-26.

Coyle, I, Sleeman, S \& Adams, N. (1995). Safety Climate. En: Journal of Safety Research, 26: 247-254.

Denison, D. (1990). Corporate Culture and Organizational Effectiveness. New York: John Wiley \& Sons.

Escobar, A, Dávila, D, Mulett, L. \& Uribe, A. (2007, Septiembre). El clima organizacional de un clúster de innovación tecnológica de la ciudad de Cali. Ponencia presentada en el III Congreso de Psicología Cognitivo Conductual, Cali, Colombia.

Espinosa, J \& Romero, L. (2002). Cuestionario de Factores Psicosociales en el Trabajo: Manual. Bogotá: HL BIO S.A.

Fernandes, M. (2008). Clima organizacional. Medidas do Comportamento Organizacional. Ferramentas de Diagnóstico e de Gestao. En: M.M. Siqueira, falta volumen: 28-38.

García, M, Rubio, P \& Bravo, L. (2007). Relación entre los factores de riesgo psicosocial y la resistencia al cambio organizacional en personal de seguridad de una empresa del sector económico terciario. En: Diversitas Perspectivas en Psicología, 3: 301-315.

Grojean, M, Resick, C, Dickson, M. \& Smith, B. (2004). Leaders, Values, and Organizational Climate: Examining Leadership Strategies for Establishing an Organizational Climate Regarding Ethics. En: Journal of Business Ethics, 55: 223-241.

Jung, D. \& Avolio, B. (2000). Opening the black box: an experimental investigation of the mediating effects of trust and value congruence on transformational and transactional leadership. En: Journal of Organizational Behavior, 21: 949-964.

King, W. (2007). A research agenda for the relationships between culture and knowledge management. En: Knowledge and process management, 14: 226-236.

Kleinman, C. (2004). Leadership and retention: Research needed. En: The Journal of Nursing Administration, 34: 111-113. Martín, J, Luceño, L, Jaén, M. \& Rubio, S. (2007). Relación entre factores psicosociales adversos, evaluados a través del cuestionario multidimensional Decore, y salud laboral deficiente. En: Psicothema, 19: 95-101. 
Medina, P. (2004). El líder y su competencia en la contención de la ansiedad grupal. En: Intangible Capital, 3: 1-8.

Meseguer, M, Soler, M, García-Izquierdo, M, Sáez, M. \& Sánchez, J. (2007). Los factores psicosociales de riesgo en el trabajo como predictores del mobbing. En: Psicothema, 19: 225-230

Niedhammer, I, Bugel, I, Goldberg, M, Leclerc, A. \& Gueguen, A. (1998). Psychosocial factors at work and sickness absence in the Gazel cohort: a prospective study. En: Occupational \& Environmental Medicine, 55: 735-741.

Pareek, U. (2004). Understanding organisational behaviour. New Delhi: Oxford University Press.

Patterson, M, West, M, Shackelton, V, Dawson, J, Lawthom, R, Maitlis, S, et al. (2005). Validating the organizational climate measure: Links to managerial practices, productivity and innovation. En: Journal of Organizational Behavior, 26: 379408.

Patterson, M, Warr, P. \& West, M (2004). Organizational climate and company performance: the role of employee affect and employee level. En: Journal of Occupational and Organizational Psychology, 77: 193-216

Peiró, J. (1995). Psicología de la Organización. Madrid: Toran S.A.

Peiró, J. (1999). El Modelo AMIGO: Marco contextualizador del desarrollo y la gestión de recursos humanos en las organizaciones.En: Papeles del psicólogo, 72, 3-15.

Pitcher, P. (1997). The drama of Leadership. New York: John Wiley and Sons.

Pitcher, P. (1999). Artists, Craftsmen \& Technocrats. En: Training \& Development, 53: 30-33.

Reichers, A \& Schneider, B. (1990). Climate and Culture: An Evolution of constructs En: Schneider, B. (Ed.) Organizational climate and culture (pp. 5-39). San Francisco: Jossey-Bass.

Schneider (Ed.). Organizational Climate and Culture. San Francisco: Qossey Bass.

Rentería, J, Fernández, E, Tenjo, A \& Uribe, A. (2009). Identifi- cación de factores psicosociales de riesgo en una empresa de producción. En: Divérsitas Perspectivas en Psicología, 5: $161-175$

Singh, A. (1998). Supervision and organizational effectiveness: Role conflict as a moderator. En: Journal of the Indian Academy of Applied Psychology, 24: 19-25.

Srivastav, A. (2005). Differential Climate in the Organization-An Empirical Study Across Functions. En: GITAM Journal of Management, 3: 90-98.

Srivastav, A. (2006). Organizational Climate as a Dependent Variable-Relationship with Role Stress, Coping Strategy and Personal Variables. En: Journal of Management Research, 6: $125-136$

Stansfeld, S, Bosman, H, Hemingway, H \& Marmot, M (1998). Psychosocial work characteristics and social support as predictors of SF-36 health functioning: The Whitehall II Study. En: Psychosomatic Medicine 60: 247-255.

Toro, F. (1998). Distinciones y relaciones entre clima, motivación, satisfacción y cultura organizacional. En: Revista Interamericana de Psicología Ocupacional, 17: 27-41.

Uribe, A. (2007). Riesgos psicosociales y productividad. Ponencia presentada en el I Congreso del Colegio Colombiano de Psicólogos. Psicología y Sociedad, Bogotá, Colombia.

Waldman, D, De Luque, M, Washburn, N, House, R, Adetoun, B, Barrasa, A. et al. (2006). Cultural and leadership predictors of corporate social responsibility values of top management: a Globe stud y of 15 countries. En: Journal of International Business Studies, 37: 823-837.

Wilkinson, A \& Wagner, R. (1993). Supervisory leadership styles and state vocational rehabilitation, counselor, job satisfaction and productivity. En: Rehabilitation Counseling Bulletin, 37: $15-24$

Zaccaro, S \& Klimoski, R. (2001). The Nature of Organizational Leadership: An Introduction. The Nature of Organizational Leadership. En: S.J. Zaccaro \& R.J. Klimoski (Eds.). (pp. 3-41). San Francisco: Qossey Bass. 